\title{
Schottky Type Ag- $\mathrm{TiO}_{2}$ Hydrogen Sensor: Gas Sensing Mechanism and Modeling
}

\author{
S. Rahbarpour ${ }^{1}$, S. M. Hosseini-Golgoo ${ }^{2}$ \\ ${ }^{1}$ Electrical Engineering Department, K. N. Toosi University of Technology, Tehran 16315-1355, Iran \\ s.rahbarpour@gmail.com \\ ${ }^{2}$ Electrical Engineering Department, Guilan University, Rasht 41635-3756, Iran
}

\begin{abstract}
The use of Schottky type noble metal-metal oxide gas sensors is spreading due to their unique gas

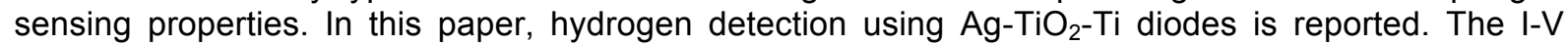
characteristics of these diodes are highly sensitive to the partial pressure of hydrogen contamination in the surrounding atmosphere. At the reducing atmosphere the energy barrier height established at the $\mathrm{Ag}-\mathrm{TiO}_{2}$ junction reduces due to silver work function variation and the junction behaves as an ohmic contact in a highly reducing atmosphere. This reversible transition from Schottky to ohmic behaviour was modelled qualitatively and quantitatively based on the hydrogen-silver interaction. The model was experimentally confirmed.
\end{abstract}

Key words: noble metal-metal oxide gas sensors, hydrogen sensor, $\mathrm{Ag}-\mathrm{TiO}_{2}$ junction, hydrogen-silver interaction, titanium oxide, Schottky type sensitivity

\section{Introduction}

Despite their many high quality factors, ceramic hydrogen sensors suffer from their limited dynamic range of sensitivity and slow response; whereas Schottky type noble metal-metal oxide hydrogen sensors show wide dynamic range of sensitivity to hydrogen along with very high and fast hydrogen response which makes them good hydrogen sensors [1,2].

The gas sensitivity of Schottky type structures is originated from both the chemical sensitivity of the metal oxide and the Schottky type sensitivity of the metal-semiconductor junction. Indeed, the origin of the extremely high atmospheric sensitivity observed in Schottky type structures has been unclear in the background literature for many years $[3,4]$. In a recent work [2], it was shown that the Schottky type sensitivity is orders of magnitude larger than chemoresistive responses and properties of Schottky type noble metal-metal oxide gas sensors are mainly defined by gas sensing properties of the utilized Schottky junction. Here, on the basis of previous work [2], hydrogen detection mechanism in $\mathrm{Ag}-\mathrm{TiO}_{2}-\mathrm{Ti}$ Schottky type diodes is shown to be microstructure dependent and is described based on the hydrogen-silver interactions. It is shown that the direct adsorption of hydrogen to the silver surface has no significant role in the hydrogen detection. Hydrogen reduces the adsorbed oxygen species on the silver surface causing extremely high variations in the obtained I-V diagram of the device. The suggested model can be applied to other noble metal-metal oxide Schottky type hydrogen sensors considering different hydrogen-metal interactions.

\section{Experiment}

$\mathrm{Ag}-\mathrm{TiO}_{2}-\mathrm{Ti}$ structures were fabricated by oxidizing titanium chips at $700{ }^{\circ} \mathrm{C}$ in air. The thickness of titanium dioxide layers were $\sim 1$ $\mu \mathrm{m}$. Residual titanium on the back side titanium oxide was used as an ohmic contact and the $\mathrm{Ag}$ contact was deposited on the other side of the oxide. This can decrease contact to contact distance which is required to minimize the chemoresistive response of the oxide in comparison to the Schottky type sensitivity of the sensor. The schematic diagram of the resulted $\mathrm{Ag}-\mathrm{TiO}_{2}-\mathrm{Ti}$ device and cross-section micrograph of the $\mathrm{TiO}_{2}$ layer grown on $\mathrm{Ti}$ substrates are depicted in Fig. $1 a$ and $b$, respectively. The micro-heater shown in Fig. 1, was positioned underneath the substrate to provide the elevated operating temperatures up to $4500^{\circ} \mathrm{C}$. The details of the fabrication technique and the measurement procedures are described elsewhere $[5,6]$. 
(a)

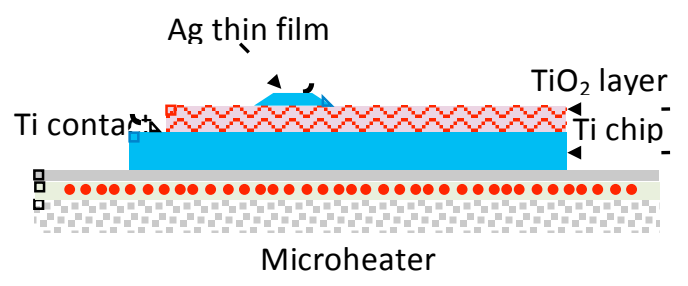

(b)

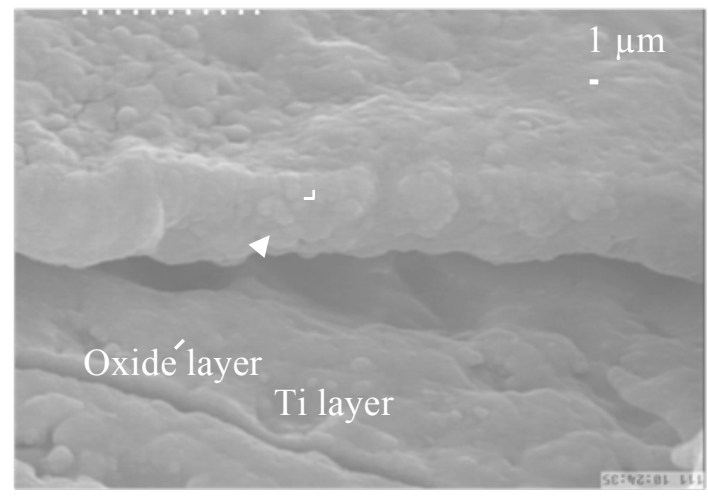

Fig. 1. The schematic diagram of the resulted $\mathrm{Ag}-\mathrm{TiO}_{2}-\mathrm{Ti}$ device (a) and cross-section SEM micrograph of the $\mathrm{TiO}_{2}$ layer grown on $\mathrm{Ti}$ substrates by thermal oxidation at $700^{\circ} \mathrm{C}$ in air (b). In (b), the oxide layer is delaminated from the titanium surface.

\section{Results and discussion}

The I-V diagrams of the fabricated $\mathrm{Ag}-\mathrm{TiO}_{2}$ diodes were studied in clean air and in pure hydrogen. The device demonstrated rectifying behavior in air while the observed Shottky barrier at $\mathrm{Ag}-\mathrm{TiO}_{2}$ junction vanishes in pure hydrogen and the device behaves linearly (Fig. $2 a \& b)$. The established barrier at the junction (1.7 eV at $\sim 330{ }^{\circ} \mathrm{C}$ in clean air) was described by considering the effect of oxygen adsorption on the $\mathrm{Ag}$ surface and hence, metal workfunction increase [5-7]. Considering two completely different behaviors of the sensor in hydrogen atmosphere and in clean air, the maximum attainable response of the sensor can be calculated by the model described below. The details are explained in ref [2].

At a constant biasing voltage and an appropriate operating temperature, the response of a sample sensor to the presence of a contaminant is defined as:

$S R=I_{g} / I_{a}$

in which $\mathrm{I}_{\mathrm{a}}$ and $\mathrm{I}_{\mathrm{g}}$ are the currents measured in clean and contaminated air, respectively. $l_{a}$ is controlled solely by the Schottky barrier height
$(\mathrm{SBH})$ and $\mathrm{I}_{\mathrm{g}}$ is related to the oxide resistance $\left(R_{s}\right)$, and hence The maximum response of the sensor can be calculated from the following equation:

$$
S R_{\max }=I_{g} / I_{a}=\frac{V / R_{s}}{A \cdot A^{*} \cdot T^{2} \cdot e^{(-\varphi / K T)}}=\frac{V}{A \cdot A^{*} \cdot T^{2} \cdot R_{s}} \cdot e^{(\varphi / K T)}
$$

where $A^{*}$ is the Richardson constant of the oxide utilized. A and $\varphi$ are the area and the $\mathrm{SBH}$ established at the junction, respectively and $V$ is the applied voltage. Using eq. (2) and the experimental data obtained from several $\mathrm{Ag}-\mathrm{TiO}_{2}$ diodes, the possibility of obtaining response levels as high as $\sim 10^{5}$ is clearly indicated. The estimated saturation response by this model is compatible with the data given in Fig. 2 which is replotted in logarithmic scale (See Fig. 3). As shown in Fig. 3, the reverse current can increase by five orders of magnitude in response to $100 \%$ hydrogen.

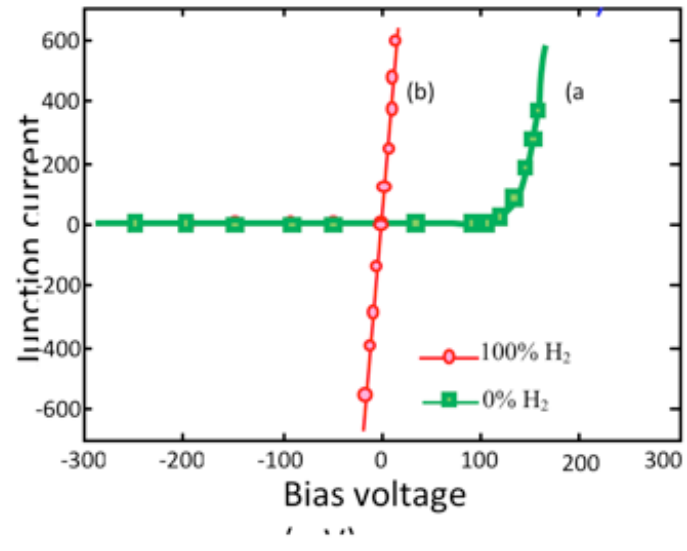

Fig. 2. The I-V diagrams obtained for a sample $\mathrm{Ag}-\mathrm{TiO}_{2}-\mathrm{Ti}$ structure in clean air (a) and in pure hydrogen (b).

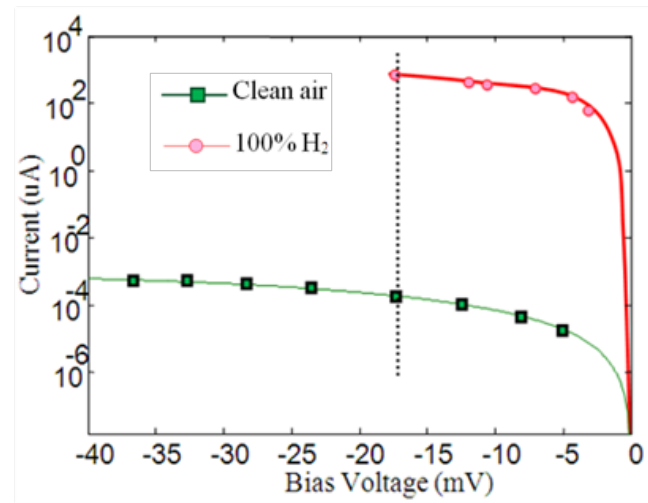

Fig. 3. The logarithmic scale presentation of reverse I-V characteristics shown in Fig. 2, demonstrating hydrogen response level as high as $10^{5}$. 


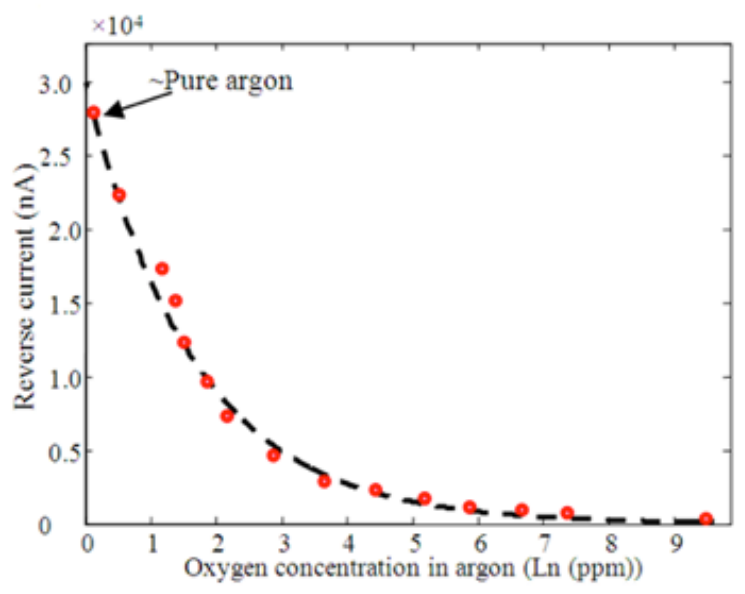

Fig. 4. The variations of the reverse current of the diode vs. oxygen concentration in argon.

Eq. (2) is also practical in all Schottky type gas sensors which demonstrate reversible transition from Schottky to ohmic behaviour at different atmospheres: Schottky type behaviour in the reference atmosphere (clean air) and linear behaviour in a highly gas contaminated atmosphere. This behaviour alteration is equal to reduction of the junction barrier to zero, regardless of interactions take place on the active surface of a gas sensor.

For example, although the hydrogen response of all noble metal- $\mathrm{TiO}_{2}$ structures are similar $[8,9]$, the gas-metal interaction occurs in Ag$\mathrm{TiO}_{2}$ structures is unique. In the case of Ag$\mathrm{TiO}_{2}-\mathrm{Ti}$ structures, barrier formation at the junction stems from oxygen adsorption on the Ag surface. Reduction of the adsorbed oxygen from the $\mathrm{Ag}$ surface, reduces the junction barrier and in a highly reducing atmosphere (such as in $\mathrm{H}_{2}$ ambient), considerable portion of the adsorbed oxygen is reduced by the gas analyte. This model predicts that long stay in pure argon, similar to $\sim 10 \mathrm{~s}$ exposure to pure hydrogen, makes the junction behave linearly. In the argon atmosphere, the adsorbed oxygen atoms are gradually desorbed and cause the barrier reduction which inturn increases the reverse current passing through the device. The concept was proved by measuring the device current in argon atmosphere (Fig. 4).

According to Fig. 4, the sensor response to pure argon, calculated by eq. (1), is $\sim 10^{5}$ which is as high as the saturation response of the sensor obtained in pure hydrogen. This means that positioning the sensor in pure argon or in pure hydrogen atmosphere are alike and cause oxygen removal from the silver surface.

In fact, the $\mathrm{Ag}-\mathrm{TiO}_{2}-\mathrm{Ti}$ structure is sensitive to the partial pressure of oxygen in its surrounding atmosphere and can be used as oxygen sensor with many desirable quality factors. It is also suitable for specific applications such as gas analyzers by integrating the sensor with a microchannel [10].

\section{Conclusion}

The I-V characteristics of $\mathrm{Ag}-\mathrm{TiO}_{2}-\mathrm{Ti}$ structures were studied in pure hydrogen and in clean air. The results showed reversible transition from Schottky to ohmic behavior. The saturation response of the sensors was both quantitatively and qualitatively modeled. It became clear that the rectifying properties of $\mathrm{Ag}-\mathrm{TiO}_{2}$ diodes are related to the oxygen adsorption on silver surface and oxygen removal from the sensor surface is the gas sensing mechanism in these sensors. Accordingly, long stay in pure argon is similar to exposure to pure hydrogen. The outcome verified by direct current measurements in both atmospheres.

\section{References}

[1] F. Hossein-Babaei, S. Abbaszadeh, M. SamieeEsfahani, Gas sensitive porous silver-rutile high temperature Schottky diode on thermally oxidized titanium, IEEE Sensors Journal 9, 237-243 (2009); doi: 10.1109/JSEN.2008.2006432.

[2] F. Hossein-Babaei, S. Rahbarpour, Separate assessment of Chemoresistivity and Schottky tpe gas sensitivity in M-metal oxide-M' structure, Sensors and Actuators B 160, 174-180 (2011); doi:10.1016/j.snb.2011.07.029

[3] T. Iwanaga, T. Hyodo, Y. Shimizu, M. Egashira, $\mathrm{H}_{2}$ sensing properties and mechanism of anodically oxidized $\mathrm{TiO}_{2}$ film contacted with $\mathrm{Pd}$ electrode, Sensors and Actuators B 93, 519-525 (2003); doi: 10.1016/S0925-4005(03)00181-3.

[4] S. K. Hazra, S. Basu, High sensitivity and fast response hydrogen sensors based in electrochemically etched porous titania thin films, Sensors and Actuators B 115, 403-411 (2006); doi: 10.1016/j.snb.2005.10.002.

[5] F. Hossein-Babaei, Porous silver- $\mathrm{TiO}_{2}$ Schottkytype chemical sensor fabricated on thermally oxidised Titanium, Electron. Lett. 44, 161-162 (2008); doi: 10.1049/el:20083269.

[6] F. Hossein-Babaei, S. Rahbarpour, Titanium and silver contacts on thermally oxidized titanium chip: Electrical and gas sensing properties, SolidState Electron. 56, 185-190 (2011); doi: 10.1016/j.sse.2010.12.007

[7] W. X. Li, C. Stampfl, M. Scheffler, Subsurface oxygen and surface oxide formation at $\mathrm{Ag}(111)$ : $\mathrm{A}$ density-functional theory investigation, Phys. Rev. B 67, 045408-045411 (2003); doi: 10.1103/PhysRevB.67.045408 
[8] S. Rahbarpour, F. Hossein-Babaei, Hydrogen detection with noble metal- $\mathrm{TiO}_{2}$ schottky diodes, Key Engineering Materials 495, 289-293 (2012); doi: 10.4028/www.scientific.net/KEM.495.289

[9] Y. Shimizu, N. Kuwano, T. Hyodo, M. Egashira, High $\mathrm{H}_{2}$ sensing performance of anodically oxidized $\mathrm{TiO}_{2}$ film contacted with $\mathrm{Pd}$, Sensors and Actuators B 83, 195-201 (2002); doi: 10.1016/S0925-4005(01)01040-1

[10] F. Hossein-Babaei, M. paknahad, V. Ghafarinia, A miniature gas analyzer made by integrating a chemoresistor with a microchannel, Lab on a Chip, in press; doi: 10.1039/C2LC00035K 